\title{
Choice and responsibility: the delegation of decision making to intelligent software agents
}

\author{
Carolyn Dowling, Paul Nicholson \\ Australian University, 115 Victoria Parade, Fitzro 3065, Victoria, Australia \\ c.dowling@patrick.acu.edu.au \\ Deakin University, 221 Burwood Highway, Burwood 3125, Victoria, Australia \\ pauln@deakin.edu.au
}

\begin{abstract}
One of the fastest growing applications of AI research is the implementation of computer programs commonly referred to as 'agents'. These programs are distinguished by a high degree of autonomy in decision making and action, the ability to 'learn' from experience and to adapt their behaviour accordingly, and often a highly personified interface. While in some cases we are aware of our interactions with these electronic entities, in many contexts their activity takes place 'behind the scenes', at a level not apparent to the user.
\end{abstract}

Implicit in the decision to make use of such programs is the assumption that they are more likely than human beings to make appropriate decisions, due to their ability to process larger amounts of information according to specific rules within a useful timeframe. When we choose to delegate this level of responsibility to computer programs it is important that we consider not simply their functional efficiency in relation to decision making, but also the broader implications of delegation, including our understandings of features of human interaction such as trust, responsibility, privacy and our capacity to judge competence and intention.

This paper explores a selection of these issues with particular references to contexts with which most computer users are familiar.

Keywords: Ethical/Ethics, Social Issues, Values, Future, Internet

NOTE: This paper describes further development of a project described in a paper presented at SECIII, Dortmund (July 200)2.

The original version of this chapter was revised: The copyright line was incorrect. This has been corrected. The Erratum to this chapter is available at DOI: 10.1007/978-0-387-35609-9_29 


\section{INTRODUCTION}

The development of intelligent software agents of varying degrees of autonomy is one of the fastest growing applications of Artificial Intelligence, and is already a well accepted aspect of our everyday computing experiences. Some agents, such as the variety of 'personalities' available to assist us with anything from the smallest to the most complex computer based task, are visible to us and to a certain extent amenable to our direct control. Others, particularly in online environments, undertake their activities at a level not apparent to the casual user.

Autonomy of decision making and action is an important criterion in distinguishing between agent software and other AI applications. 'Autonomy', within these contexts, means that the program is able to make decisions and initiate actions without the need for constant monitoring and intervention on the part of users. Much of the capacity of such programs to act independently derives from their ability to 'learn' from experience and thus to respond flexibly to changing situations, perhaps in ways not clearly predicted by the original programmers. A highly personified interface suggesting the possession of a believable 'character' or personality provides an important basis for the agent's interaction with users, including a propensity for users to be comfortable with delegating decision making to these entities.

Early definitions of agent software, based more on speculation than on experience, tended to emphasise the broader 'human' aspects of these programs. Laurel, for instance, describes an agent as "A character, enacted by the computer, who acts on behalf of the user in a virtual environment", useful in mediating " ... a relationship between the labyrinthine precision of computers and the fuzzy complexity of man [3, p. 355]. Typical of more functional definitions is: "An agent can be viewed as an object which has a goal and autonomously solves problems through interaction, such as collaboration, competition, negotiation and so on" [2]. From another perspective, an agent exhibiting these characteristics can be described as having, " a set of beliefs about its environment and about itself; a set of desires which are computational states which it wants to maintain, and a set of intentions which are computational states which the agent is trying to achieve" [6].

Current interest in agent based systems in a range of areas raises a number of issues in relation to the decision-making roles which we delegate to them in areas of significant importance to human activity and wellbeing such as medicine, finance and defence. They include the balance between autonomy and control, and questions related to trust, responsibility and 
privacy. These areas of concern relate also to more commonplace applications, in particularly those associated with the Internet.

\section{SOME FEATURES OF THE 'AGENT' METAPHOR}

Why have we so readily accepted the concept of software agents as an essentially unproblematic element in our everyday computing environments? Firstly, they fulfill a need. An increasing number of our day to day activities now take place within computing environments characterised by rapid change, large quantities of extraordinarily complex information, and a lack of common organisational structures through which information may be accessed and managed. As Laurel [3] predicted, there are now many situations in which some form of 'intelligent' mediation is required between computer systems and the needs of users.

Secondly, as an interface metaphor, the notion of an 'agent' strikes a comforting note of familiarity with many computer users, most commonly conjuring up an image of a compliant servant undertaking various tasks at the behest and in the interests of the user. As a way of conceptualising our relationship with the technology it has a great deal in common with an earlier metaphor which proved extremely popular, that of the computer as a 'tool'. Both of these images incorporate the reasssuring suggestion that the technology is under the control of the user. In each case, however, the metaphor is less straightforward than it appears, and the confidence and security engendered in users may well be misplaced. The notion of an agent may mask not just a functional complexity, but also a range of activities that are not of users' choosing, and not necessarily even in their interest. An obvious example is the proliferation of agents specializing in the compilation of profiles of visitors to Internet sites to be used for marketing purposes.

In conceptualizing these software entities in a 'compliant servant' role, we often forget that our experiences of 'agents' in the physical world are actually quite varied, and this may be reflected in the nature and activities of their electronic counterparts. Some of the most interesting types of human 'agents' to consider in relation to the delegation of decision making responsibilities to their software equivalents are those which actually represent the interests of more than one party, and must effect a balance between competing claims. Examples of these in the physical world include estate agents, theatrical or literary agents, and employment agents.

Another type of agent with which we are all familiar but are generally less comfortable, is the 'secret agent', usually a gatherer of supposedly confidential information operating under an assumed identity. This model is particularly relevant to some current practices on the Internet. Far from 
being initiated through a contractual arrangement entered into voluntarily by the user, interaction with many such agents is an invisible and frequently unsought component of an apparently innocuous task such as a search for information.

Common to all of the understandings of the roles and functions of human agents outlined above is the assumption that their usefulness derives from the possession of specialised skills, which qualify them to mediate between an individual and a particular environment for the more effective achievement of various ends. Furthermore, there exists a commonly held understanding that most human agents can be relied upon to act at least to a certain extent in accordance with an understood set of ethical principles that ensure that the client or user is not disadvantaged. Such assumptions probably play a significant part in encouraging the acceptance of programs characterised as 'agents'.

\section{THE ROLE OF PERSONIFICATION}

Where the user is intended to be aware of the activities of the software and to interact knowingly with it, the agent metaphor lends itself particularly well to reinforcement through a personified interface. Anthropomorphic elements have been implicit in most computer interfaces from the earliest days. Intelligence and language use are widely accepted as the key criteria distinguishing us both from other living things and from inanimate objects. The use of the term 'intelligence', albeit 'artificial', in relation to computer programs, in combination with the fact that for the most part we interact with them through language, adds support to the perception that a software agent is 'one of us', and should be subject to the types of expectations, including that of trust, that govern our interactions with our fellow human beings.

The usefulness of a significantly personified interface in promoting trust between user and agent is complicated, however, by the fact that both research and experience suggest that a mismatch between realism in appearance and the apparent knowledge level of the agent as revealed through its use of language and other capabilities can have a deleterious effect on credibility and on acceptance. As Masterton, writes, "A common problem with AI programs that interact with humans is that they must present themselves in a way that reflects their ability. Where there is a conflict between the ability of the system and the users' perception of that ability a breakdown occurs and users may either fail to exploit its full potential or become frustrated with its shortcomings" [4, p. 215]. Agents that 'look' smart and 'act' or 'talk' dumb are poorly received by many users, who express a higher tolerance for the limitations of a 'character' more 
sketchily represented, for instance through cartoon-like graphics, even one characterised as a normally inanimate object or an animal [1]. This may provide some counterbalance to our tendency to unquestioningly invest a high level of trust in such entities. Here too, however, there are complexities, although of a psychological rather than an ethical nature. While in many instances we may feel more comfortable giving instructions to a 'character' that is not fully human, we may not feel the same way about receiving advice or even correction from such an entity. Not all users of Microsoft Office are comfortable with being subjected to the whims of an animated paperclip.

The issue of the degree to which software agents can and should be personified is extremely important when considering any ethical aspects of agent systems. It is this aspect of agency that propels questions of right and wrong to a level beyond that pertaining to our use of more traditional types of programs. To the extent that personification is successfully implemented, the expectations of users in relation to the behaviour of the program move into the arena governing the understandings upon which we base our interpersonal interactions, rather than merely those upon which we base our use of technology. The problems are accentuated in cases where the agent emulates human dialogue through text or 'voice' technology with such success that users may not be aware that they are interacting with a computer program. This occurs quite frequently in real-time interactive environments such as chat room and MOOs. Such a situation is inherently and deliberately misleading, and falls short of being an ideal basis for the development of the type of trust in agency that arguably should be an important part of a fruitful ongoing relationship between human beings and software agents.

\section{SOME ISSUES ARISING FROM THE DELEGATION OF DECISION MAKING TO AGENTS}

The delegation of any task to a software agent raises questions in relation not only to trust but also to its autonomy of action and decision, and to the location of responsibility, both moral and legal, for the outcomes of those decisions and actions.

Programs of this type are being developed specifically to weigh up complex information, make decisions and initiate actions in 'mission critical' areas of human endeavour including government, health, scientific research, commerce, the law and defence, with a significant degree of autonomy being intrinsic to their usefulness. Implicit in the recognition of the need for a capacity to exercise initiative is an acknowledgment that some outcomes of agent activity may not be easily predictable by the user. In some 
cases, as mentioned earlier, they may even be contrary to what the user might perceive as his or her interests and wishes.

A further issue emanating from consideration of agent autonomy is that of responsibility for outcomes and actions resulting from decisions which are out of the user's control, and which may indeed relate to capacities of the program of which the user had no knowledge at all. It is inevitable that such instances will occur, precipitating the need for renewed examination both of community and legal understandings of liability.

The issue of trust, as discussed earlier in relation to personification, is critical to the delegation of responsibility, whether to a human or to an electronic agent. How do we decide whether or not to 'trust' an agent? In the physical world a reputation for credibility in most fields is commonly contingent on a verifiable history demonstrating qualities in relation to the field of expertise such as accuracy, reliability, efficiency and so on. In addition we often rely on the recommendation of other 'trusted' persons. Where such assurances are available in relation to the software agents to whom we delegate responsibility, perhaps we can be justified in taking the risk of 'trusting' them. As with some forms of delegation to other human beings, however, we may in fact have to accept that there are no absolute guarantees, and that acceptance of some degree of risk is an ever-present element in the act of delegation.

Even if we are prepared to trust the agents with whom we deal directly, a further question remains as to how agents might reasonably 'decide' how to trust one another. Implications for users include important considerations in regard to privacy. Van der Lubbe [7, p. 223], for instance, defines the main threats to privacy as "Threats cause by agents acting on behalf of the user (through loss of control of the user on (sic) his agent and the disclosure of the user's personal information)" and "Threats caused by foreign agents that act on behalf of others (information extraction via traffic flow monitoring, data mining and even covert attempts to obtain personal information directly from the user's agent ...)".

\section{ETHICS AND AGENCY}

As noted earlier, an important element in our decisions to invest trust in expert human agents is our belief that their actions will be governed by a code or codes of ethical behaviour, both at a personal and a professional level. Adherence to a code of ethics is, in fact, a key element in the definition of a profession.

At a purely conceptual level, regardless of issues of implementation, what might a code of ethics for software agents look like? Given that agents 
could well be described as the disembodied 'robots' of cyberspace, an appropriate place to start might be Isaac Asimov's Three Laws of Robotics, familiar to several generations of science fiction readers. A generalised version of the original laws is the directive that a robot may not injure humanity or, through inaction, allow humanity to come to harm.

AI researcher Marvin Minsky acknowledges issues similar to those recognised by Asimov in relation specifically to software agents when he writes: "There's the old paradox of having a very smart slave. If you keep the slave from learning too much, you are limiting its usefulness. But, if you help it to become smarter than you are, then you may not be able to trust it not to make better plans for itself than it does for you" [5, p. 25]. Some current research in this area expresses the problem in terms of the need for the types of 'norms' that constrain behaviour within human society [8].

\section{QUERYING AN UNDERLYING ASSUMPTION}

An implicit element in the implementation of agent systems is the assumption that decisions deriving from consideration of the greatest possible number of relevant factors must necessarily be the most efficacious. While the computer's superiority over human beings in processing large amounts of information is undeniable, we should not lose sight of the fact that this data is manipulated according to rules formulated by human beings, whose understanding presumably suffers from the very deficiencies in data handling capacity that make the prospect of decision making by computer so attractive. Further, current thinking in relation to human decision making is less clear on the question of whether or not the consideration of more data inevitably leads to a 'better' decision.

\section{CONCLUSION}

The use of intelligent software possessing a significant degree of autonomy is here to stay, and its usefulness in many areas is clear. What is less clear is where the balance should lie between the use of these technologies and the desirability of involving the 'human factor' in decision making. These concerns are not new in relation to AI, but have gained fresh urgency with the increase in actual implementation of this type of software. While to a certain extent our choice to delegate responsibility for decisionmaking to software agents can be based on criteria similar to those we apply to human agents, there are a number of areas that need clarification including 
standards of ethical behaviour and legal and moral responsibility for the outcome of decisions taken. Of particular concern is the fact that we are not always given the choice, as it is increasingly common for agent software to operate in the 'background' areas of an application. This is an area where public awareness should be increased so as to stimulate more dialogue between systems developers, users, ethicist and even lawyers.

\section{REFERENCES:}

1. Chan, T.-W. (1998), 'The past, present, and future of educational agents', http://www.apc.src.ncu.edu.tw/apc/ppt chan.html

2. Kinoshita T., Sugawara K. (1995) Agent oriented computing Tokyo Soft Research Center.

3. Laurel, B. (1990), 'Interface agents: metaphors with character', In Laurel, B. (ed.) The Art of Human-Computer Interface Design, Reading, Massachusetts: Addison-Wesley.

4. Masterton, S. (1998), 'Computer support for learners using intelligent educational agents: the way forward', in Global Education on the Net Vol. I, (Chan T.-W., Collins A., Lin J. eds), Proc.ICCE '98, the Sixth International Conference on Computers in Education, Beijing, China, October 1998, Beijing, China Higher Education Press \& Springer-Verlag

5. Minsky, M. (1994), 'A conversation with Marvin Minsky about Agents', Communications of the ACM, July 1994, vol. 37, no. 7 .

6. O'Hare, G. (2000), 'Agency, Mobility and Virtuality: A Necessary Synergy', in Proc. ISA '2000, Internatl ICSC Congress on Intelligent Systems and Applications, Wollongong, Australia, 11 - 15 December 2000, Canada: ICSC Academic Press, pp. 15 - 21.

7. van der Lubbe, J. (2000), 'Incorporating Privacy Enhancing Technology in Intelligent Software Agents', in Proceedings of ISA '2000, International ICSC Congress on Intelligent Systems and Applications, Wollongong, Australia, 11 - 15 December 2000, Canada: ICSC Academic Press, pp. 222 - 228.

8. Verhagen, H. (2000), 'Trust and Norms for Artificial Agents', in Proceedings of ISA '2000, International ICSC Congress on Intelligent Systems and Applications, Wollongong, Australia, December 2000, Canada: ICSC Academic Press, pp. 277 - 280. 